\title{
A New Formulation to the Point Kinetics Equations Considering the Time Variation of the Neutron Currents
}

\author{
Anderson Lupo Nunes ${ }^{1}$, Aquilino Senra Martinez ${ }^{1}$, Fernando Carvalho da Silva ${ }^{1}$, \\ Daniel Artur Pinheiro Palma ${ }^{2}$ \\ ${ }^{1}$ Department of Nuclear Engineering, COPPE/UFRJ, Rio de Janeiro, Brazil \\ ${ }^{2}$ Brazilian Commission for Nuclear Energy, Rio de Janeiro, Brazil \\ Email: anunes@con.ufri.br, aquilino@Imp.ufri.br, fernando@con.ufri.br, dpalmaster@gmail.com
}

Received 6 January 2015; accepted 27 January 2015; published 29 January 2015

Copyright @ 2015 by authors and Scientific Research Publishing Inc.

This work is licensed under the Creative Commons Attribution International License (CC BY).

http://creativecommons.org/licenses/by/4.0/

(c) (i) Open Access

\section{Abstract}

The system of point kinetics equations describes the time behaviour of a nuclear reactor, assuming that, during the transient, the spatial form of the flux of neutrons varies very little. This system has been largely used in the analysis of transients, where the numerical solutions of the equations are limited by the stiffness problem that results from the different time scales of the instantaneous and delayed neutrons. Its derivation can be done directly from the neutron transport equation, from the neutron diffusion equation or through a heuristics procedure. All of them lead to the same functional form of the system of differential equations for point kinetics, but with different coefficients. However, the solution of the neutron transport equation is of little practical use as it requires the change of the existent core design systems, as used to calculate the design of the cores of nuclear reactors for different operating cycles. Several approximations can be made for the said derivation. One of them consists of disregarding the time derivative for neutron density in comparison with the remaining terms of the equation resulting from the P1 approximation of the transport equation. In this paper, we consider that the time derivative for neutron current density is not negligible in the P1 equation. Thus being, we obtained a new system of equations of point kinetics that we named as modified. The innovation of the method presented in the manuscript consists in adopting arising from the P1 equations, without neglecting the derivative of the current neutrons, to derive the modified point kinetics equations instead of adopting the Fick's law which results in the classic point kinetics equations. The results of the comparison between the point kinetics equations, modified and classical, indicate that the time derivative for the neutron current density should not be disregarded in several of transient analysis situations. 


\section{Keywords}

\section{Reactor Point-Kinetics, Neutron Current Density, Nuclear Power Density}

\section{Introduction}

In order to determine the nuclear power distribution in a reactor core, one should investigate the neutron transport in a heterogeneous medium and with strong neutron absorption, where these neutrons can also be scattered or escape from the active part of the reactor. Notwithstanding that, the advances in computer processing and of the countless methods to solve the neutron transport equation, in practice the approximation of the neutron diffusion is largely used in stationary calculations to predict the distribution of neutrons and of the critical concentration of boron. To deal with the movement of the neutrons in a way similar to that of heat diffusion, one needs to make several approximations in the transport equation, which include a weak angular dependency of the angular distribution of the neutrons, isotropic sources of neutrons, and the disregarding of the derivative for neutron current density, in comparison with other terms that appear in the neutron transport equation [1].

Once the spatial distribution of the neutrons in the nuclear reactor is known, it is also important to predict the time behaviour of this distribution, induced as it is by the variation in nuclear reactivity due to the variation of fuel temperature, the variation of the material composition of the reactor core, the variation in moderator density, amongst others. The simplest way to determine the time behaviour for the nuclear power is through the solution of point kinetics equations. These equations include approximations that are added to those made to obtain the equations for neutron diffusion in the structure of multi-groups of neutron energy [2]. Point kinetics equations consist on a system for the calculation of the nuclear power and the concentrations of the delayed neutrons precursors. They are first-order differential equations, coupled and non-linear in their more general form.

Though quite questionable, the approximations made in the development of the classical point kinetics equations have already been widely analysed and discussed in the literature. However, the influence on these equations from not considering the time derivative in neutron current density did not deserve, until now, a systematic and specific evaluation. Due to this, in this paper we developed, from the neutron transport equation, the modified point kinetics equations, that are different from the classical ones, as they include the time derivative of neutron current density.

In Section 2, it is presented the development of the modified point kinetics equations. Section 3 presents the calculation to obtain their analytical solutions. Section 4 presents the results of the analytical solutions of the classical and modified point kinetics equations. And Section 5 discusses the results obtained and provides the conclusions of this paper.

\section{The Modified Point Kinetics Equations}

The theory of neutron transport is the wide model to describe neutron distribution in a nuclear reactor. It is described in [1] and [3] in terms of the angular flux of neutrons, $\varphi(\boldsymbol{r}, E, \hat{\Omega}, t)$ :

$$
\frac{1}{v(E)} \frac{\partial \varphi(\boldsymbol{r}, E, \hat{\Omega}, t)}{\partial t}+L_{1} \varphi(\boldsymbol{r}, E, \hat{\Omega}, t)=F_{1} \varphi(\boldsymbol{r}, E, \hat{\Omega}, t)+\frac{1}{4 \pi} \sum_{i=1}^{6} \lambda_{i} \chi_{i}(E) C_{i}(\boldsymbol{r}, t)-\sum_{i=1}^{6} F_{i 1} \varphi(\boldsymbol{r}, E, \hat{\Omega}, t)
$$

and

$$
\frac{1}{4 \pi} \chi_{i}(E) \frac{\partial C_{i}(\boldsymbol{r}, t)}{\partial t}=F_{i 1} \varphi\left(\boldsymbol{r}, E^{\prime}, \hat{\Omega}^{\prime}, t\right)-\frac{1}{4 \pi} \lambda_{i} \chi_{i}(E) C_{i}(\boldsymbol{r}, t)
$$

where $i=1,2, \cdots, 6$ and the operators $L_{1}, F_{1}, F_{p 1}$ and $F_{i 1}$ are defined as follows:

$$
\begin{gathered}
L_{1}(\cdot) \equiv \hat{\Omega} \cdot \nabla(\cdot)+\Sigma_{t}(\boldsymbol{r}, E, t)-\int_{4 \pi}^{\infty} \int_{0}^{\infty} \Sigma_{S}\left(\boldsymbol{r}, E^{\prime} \rightarrow E, \hat{\Omega}^{\prime} \rightarrow \hat{\Omega}, t\right)(\cdot) \mathrm{d} E^{\prime} \mathrm{d} \hat{\Omega}^{\prime}, \\
F_{1}(\cdot) \equiv F_{p 1}(\cdot)+\sum_{i=1}^{6} F_{i 1}(\cdot),
\end{gathered}
$$




$$
F_{p 1}(\cdot) \equiv \frac{1}{4 \pi}(1-\beta) \chi_{f}(E) \iint_{4 \pi}^{\infty} v(E) \Sigma_{f}\left(\boldsymbol{r}, E^{\prime}, t\right)(\cdot) \mathrm{d} E^{\prime} \mathrm{d} \hat{\Omega}^{\prime}
$$

and

$$
F_{i 1}(\cdot) \equiv \frac{1}{4 \pi} \beta_{i} \chi_{i}(E) \iint_{4 \pi}^{\infty} v\left(E^{\prime}\right) \Sigma_{f}\left(\boldsymbol{r}, E^{\prime}, t\right)(\cdot) \mathrm{d} E^{\prime} \mathrm{d} \hat{\Omega}^{\prime} .
$$

The scattering cross section can be expanded in terms of the polynomials of Legendre up to the second term, that is, the expansion is done for $l=0$ and $l=1$. It consists of the $P_{1}$ approximation

$$
\Sigma_{S}\left(\boldsymbol{r}, E^{\prime} \rightarrow E, \hat{\Omega}^{\prime} \rightarrow \hat{\Omega}, t\right) \cong \sum_{l=0}^{1} \frac{2 l+1}{4 \pi} \Sigma_{S l}\left(\boldsymbol{r}, E^{\prime} \rightarrow E, t\right) P_{l}\left(\hat{\Omega}^{\prime} \cdot \hat{\Omega}\right) .
$$

Equation (3) can be re-written thus:

$$
L_{1}(\cdot) \equiv \hat{\Omega} \cdot \nabla(\cdot)+\Sigma_{t}(\boldsymbol{r}, E, t)-\int_{4 \pi}^{\infty} \int_{0}^{\infty} \frac{1}{4 \pi} \Sigma_{S 0}\left(\boldsymbol{r}, E^{\prime} \rightarrow E, t\right)(\cdot) \mathrm{d} E^{\prime} \mathrm{d} \hat{\Omega}^{\prime}-\int_{4 \pi}^{\infty} \int_{0}^{\infty} \frac{3}{4 \pi} \Sigma_{S 1}\left(\boldsymbol{r}, E^{\prime} \rightarrow E, t\right) \hat{\Omega^{\prime}} \cdot \hat{\Omega}(\cdot) \mathrm{d} E^{\prime} \mathrm{d} \hat{\Omega}^{\prime}(8)
$$

We apply the operator $\int_{4 \pi}(\cdot) \mathrm{d} \hat{\Omega}$ to Equations (1) and (2) and considering the following definitions:

$$
\phi(\boldsymbol{r}, E, t) \equiv \int_{4 \pi} \varphi(\boldsymbol{r}, E, \hat{\Omega}, t) \mathrm{d} \hat{\Omega}
$$

and

$$
\boldsymbol{J}(\boldsymbol{r}, E, t) \equiv \int_{4 \pi} \varphi(\boldsymbol{r}, E, \hat{\Omega}, t) \hat{\Omega} \mathrm{d} \hat{\Omega}
$$

it results that:

$$
\frac{1}{v(E)} \frac{\partial \phi(\boldsymbol{r}, E, t)}{\partial t}+\nabla \cdot \boldsymbol{J}(\boldsymbol{r}, E, t)+L \phi(\boldsymbol{r}, E, t)=F \phi(\boldsymbol{r}, E, t)+\sum_{i=1}^{6} \lambda_{i} \chi_{i}(E) C_{i}(\boldsymbol{r}, t)-\sum_{i=1}^{6} F_{i} \phi(\boldsymbol{r}, E, t)
$$

and

$$
\chi_{i}(E) \frac{\partial C_{i}(\boldsymbol{r}, t)}{\partial t}=F_{i} \phi(\boldsymbol{r}, E, t)-\lambda_{i} \chi_{i}(E) C_{i}(\boldsymbol{r}, t)
$$

where the operators $L, F$ and $F_{i}$ are defined:

$$
\begin{aligned}
& L(\cdot) \equiv \Sigma_{T}(\boldsymbol{r}, E, t)-\int_{0}^{\infty} \Sigma_{S 0}\left(\boldsymbol{r}, E^{\prime} \rightarrow E, t\right)(\cdot) \mathrm{d} E^{\prime}, \\
& F(\cdot)=\left\{(1-\beta) \chi_{f}(E)+\sum_{i=1}^{6} \beta_{i} \chi_{i}(E)\right\} \int_{0}^{\infty} v\left(E^{\prime}\right) \Sigma_{f}\left(\boldsymbol{r}, E^{\prime}, t\right)(\cdot) \mathrm{d} E^{\prime}
\end{aligned}
$$

and

$$
F_{i}(\cdot) \equiv \beta_{i} \chi_{i}(E) \int_{0}^{\infty} v\left(E^{\prime}\right) \Sigma_{f}\left(\boldsymbol{r}, E^{\prime}, t\right)(\cdot) \mathrm{d} E^{\prime} .
$$

In replacing Equation (8) in Equation (1), multiplying the resulting equation by $\Omega$ and after that integrating in the solid angle, it results that:

$$
\frac{1}{v(E)} \frac{\partial \boldsymbol{J}(\boldsymbol{r}, E, t)}{\partial t}+\frac{1}{3} \nabla \phi(\boldsymbol{r}, E, t)+\Sigma_{t}(\boldsymbol{r}, E, t) \boldsymbol{J}(\boldsymbol{r}, E, t)=\int_{0}^{\infty} \Sigma_{S 1}\left(\boldsymbol{r}, E^{\prime} \rightarrow E, t\right) \boldsymbol{J}\left(\boldsymbol{r}, E^{\prime}, t\right) \mathrm{d} E^{\prime}
$$

Considering the approximation described in [1]:

$$
\Sigma_{S 1}\left(\boldsymbol{r}, E^{\prime} \rightarrow E, t\right) \cong \Sigma_{S 1}\left(\boldsymbol{r}, E^{\prime}, t\right) \delta\left(E^{\prime}-E\right)
$$

and defining the transport cross section: 


$$
\Sigma_{t r}(\boldsymbol{r}, E, t) \equiv \Sigma_{t}(\boldsymbol{r}, E, t)-\Sigma_{S 1}(\boldsymbol{r}, E, t),
$$

from Equation (14), it is possible to write:

$$
\frac{1}{v(E)} \frac{\partial \boldsymbol{J}(\boldsymbol{r}, E, t)}{\partial t}+\Sigma_{t r}(\boldsymbol{r}, E, t) \boldsymbol{J}(\boldsymbol{r}, E, t)=-\frac{1}{3} \nabla \phi(\boldsymbol{r}, E, t)
$$

There is a similarity of Equation (15) with the Telegrapher Equation, as can be seen in [4] and [5]. However the methodology used to arrive at Equation (15) is totally different, as well as the results that follow until we get to the Equation (28).

In dividing Equation (15) by the transport cross section, using the definition of diffusion coefficient, applying the diverging operator and disregarding the term $\nabla D \cdot \frac{\partial J}{\partial t}$, it results that:

$$
\frac{3 D(\boldsymbol{r}, E, t)}{v(E)} \frac{\partial \boldsymbol{\nabla} \cdot \boldsymbol{J}(\boldsymbol{r}, E, t)}{\partial t}+\nabla \cdot \boldsymbol{J}(\boldsymbol{r}, E, t)=-\nabla \cdot[D(\boldsymbol{r}, E, t) \nabla \phi(\boldsymbol{r}, E, t)],
$$

where the diffusion coefficient is defined as:

$$
D(\boldsymbol{r}, E, t) \equiv \frac{1}{3 \Sigma_{t r}(\boldsymbol{r}, E, t)} .
$$

After that, in deriving Equation (9) in relation to time, and multiplying by $\frac{3 D(\boldsymbol{r}, E, t)}{v(E)}$, and adding to the very Equation (9) and replacing Equation (16), one obtains:

$$
\begin{aligned}
& \frac{3 D(r, E, t)}{(v(E))^{2}} \frac{\partial^{2} \phi(\boldsymbol{r}, E, t)}{\partial t^{2}}-\nabla(D(\boldsymbol{r}, E, t) \nabla \phi(\boldsymbol{r}, E, t))+\frac{1}{v(E)} \frac{\partial \phi(\boldsymbol{r}, E, t)}{\partial t}+L \phi(\boldsymbol{r}, E, t) \\
& \quad+\frac{3 D(\boldsymbol{r}, E, t)}{v(E)} \frac{\partial}{\partial t}\{(L-F) \phi(\boldsymbol{r}, E, t)\} \\
& =\sum_{i=1}^{6} \lambda_{i} \chi_{i}(E) \frac{3 D(\boldsymbol{r}, E, t)}{v(E)} \frac{\partial C_{i}(\boldsymbol{r}, t)}{\partial t}+F \phi(\boldsymbol{r}, E, t)-\sum_{i=1}^{6} \frac{3 D(\boldsymbol{r}, E, t)}{v(E)} \frac{\partial}{\partial t}\left\{F_{i} \phi(\boldsymbol{r}, E, t)\right\} \\
& \quad+\sum_{i=1}^{6} \lambda_{i} \chi_{i}(E) C_{i}(\boldsymbol{r}, t)-\sum_{i=1}^{6} F_{i} \phi(\boldsymbol{r}, E, t) .
\end{aligned}
$$

In the Equations (10) and (17), when in a stationary regime, all of its time derivatives are disregarded. Then, it results that:

$$
-\nabla\left(D\left(\boldsymbol{r}, E, t_{0}\right) \nabla \phi\left(\boldsymbol{r}, E, t_{0}\right)\right)+\left(L_{0}-F_{0}\right) \phi\left(\boldsymbol{r}, E, t_{0}\right)=0,
$$

Considering the adjoint flux of neutrons from Equation (18), this adjoint equation is:

$$
-\nabla \cdot\left(D\left(\boldsymbol{r}, E, t_{0}\right) \nabla \phi^{*}\left(\boldsymbol{r}, E, t_{0}\right)\right)+\left(L_{0}^{+}-F_{0}^{+}\right) \phi^{*}\left(\boldsymbol{r}, E, t_{0}\right)=0 .
$$

where,

$$
\begin{gathered}
L_{0}^{+}(\cdot) \equiv \Sigma_{t}\left(\boldsymbol{r}, E, t_{0}\right)-\int_{0}^{\infty} \Sigma_{s 0}\left(\boldsymbol{r}, E \rightarrow E^{\prime}, t_{0}\right)(\cdot) \mathrm{d} E^{\prime} \\
F_{0}^{+}(\cdot) \equiv(1-\beta) v(E) \Sigma_{f}\left(\boldsymbol{r}, E, t_{0}\right) \int_{0}^{\infty} \chi_{f}\left(E^{\prime}\right)(\cdot) \mathrm{d} E^{\prime}+\sum_{i=1}^{6} \beta_{i} v(E) \Sigma_{f}\left(\boldsymbol{r}, E, t_{0}\right) \int_{0}^{\infty} \chi_{i}\left(E^{\prime}\right)(\cdot) \mathrm{d} E^{\prime} \\
L_{0}(\cdot) \equiv \Sigma_{t}\left(\boldsymbol{r}, E, t_{0}\right)-\int_{0}^{\infty} \Sigma_{s 0}\left(\boldsymbol{r}, E^{\prime} \rightarrow E, t_{0}\right)(\cdot) \mathrm{d} E^{\prime}
\end{gathered}
$$


and

$$
F_{0}(\cdot) \equiv(1-\beta) \chi_{f}(E) \int_{0}^{\infty} v\left(E^{\prime}\right) \Sigma_{f}\left(\boldsymbol{r}, E^{\prime}, t_{0}\right)(\cdot) \mathrm{d} E^{\prime}+\sum_{i=1}^{6} \beta_{i} \chi_{i}(E) \int_{0}^{\infty} v\left(E^{\prime}\right) \Sigma_{f}\left(\boldsymbol{r}, E^{\prime}, t_{0}\right)(\cdot) \mathrm{d} E^{\prime}
$$

Equation (18) is integrated in the volume and in the energy, and re-written:

$$
-\int_{V}^{\infty} \int_{0}^{\infty} \phi^{*}\left(\boldsymbol{r}, E, t_{0}\right) \nabla\left(D\left(\boldsymbol{r}, E, t_{0}\right) \nabla \phi(\boldsymbol{r}, E, t)\right) \mathrm{d} E \mathrm{~d}^{3} r+\int_{V}^{\infty} \int_{0}^{\infty} \phi^{*}\left(\boldsymbol{r}, E, t_{0}\right)\left(L_{0}-F_{0}\right) \phi(\boldsymbol{r}, E, t) \mathrm{d} E \mathrm{~d}^{3} r=0
$$

In multiplying Equation (17) by the adjoint flux of neutrons and integrating in the volume and in the energy $E$, and subtracting of the result by Equation (24), one obtains:

$$
\begin{aligned}
& \int_{V}^{\infty} \int_{0}^{\infty} \frac{3 D}{v^{2}} \phi^{*}\left(\boldsymbol{r}, E, t_{0}\right) \frac{\partial^{2} \phi(\boldsymbol{r}, E, t)}{\partial t^{2}} \mathrm{~d} E \mathrm{~d}^{3} r-\int_{V}^{\infty} \int_{0}^{\infty} \phi^{*}\left(\boldsymbol{r}, E, t_{0}\right)\left(L_{0}-F_{0}\right) \phi(\boldsymbol{r}, E, t) \mathrm{d} E \mathrm{~d}^{3} r \\
& \quad+\int_{V}^{\infty} \int_{0}^{\infty} \frac{1}{V} \phi^{*}\left(\boldsymbol{r}, E, t_{0}\right) \frac{\partial \phi(\boldsymbol{r}, E, t)}{\partial t} \mathrm{~d} E \mathrm{~d}^{3} r+\int_{V}^{\infty} \int_{0}^{\infty} \frac{3 D}{V} \phi^{*}\left(\boldsymbol{r}, E, t_{0}\right) \frac{\partial}{\partial t}\{(L-F) \phi(\boldsymbol{r}, E, t)\} \mathrm{d} E \mathrm{~d}^{3} r \\
& \quad+\int_{V}^{\infty} \int_{0}^{\infty} \phi^{*}\left(\boldsymbol{r}, E, t_{0}\right)(L-F) \phi(\boldsymbol{r}, E, t) \mathrm{d} E \mathrm{~d}^{3} r \\
& =\sum_{i=1}^{6} \int_{V}^{\infty} \int_{0}^{\infty} \lambda_{i} \chi_{i}(E) \phi^{*}\left(\boldsymbol{r}, E, t_{0}\right) \frac{3 D}{V} \frac{\partial C_{i}(\boldsymbol{r}, t)}{\partial t} \mathrm{~d} E \mathrm{~d}^{3} r-\sum_{i=1}^{6} \int_{V} \int_{0}^{\infty} \frac{3 D}{V} \phi^{*}\left(\boldsymbol{r}, E, t_{0}\right) \frac{\partial}{\partial t}\left\{F_{i} \phi(\boldsymbol{r}, E, t)\right\} \mathrm{d}^{2} \mathrm{~d}^{3} r \\
& \quad+\sum_{i=1}^{6} \int_{V}^{\infty} \int_{0}^{\infty} \lambda_{i} \chi_{i}(E) \phi^{*}\left(\boldsymbol{r}, E, t_{0}\right) C_{i}(\boldsymbol{r}, t) \mathrm{d} E \mathrm{~d}^{3} r-\sum_{i=1}^{6} \int_{V}^{\infty} \int_{0}^{*} \phi^{*}\left(\boldsymbol{r}, E, t_{0}\right) F_{i} \phi(\boldsymbol{r}, E, t) \mathrm{d} E \mathrm{~d}^{3} r,
\end{aligned}
$$

where, by simplicity, the functional dependence both of the diffusion coefficient as of the speed was omitted. The neutron flux is written as the product of an amplitude factor $n(t)$, which is dependent on time only, and a shape factor (or shape function) $f(\boldsymbol{r}, E)$; thus,

$$
\phi(\boldsymbol{r}, E, t) \cong n(t) f(\boldsymbol{r}, E) .
$$

In writing the neutron flux as the product of the two factors in Equation (26), the intent is that the amplitude factor, $n(t)$, should describe most of the time dependence whereas the shape factor, $f(\boldsymbol{r}, E)$, will change very little with time.

In defining the integral:

$$
I_{F} \equiv \int_{V} \int_{0}^{\infty} \phi^{*}\left(\boldsymbol{r}, E, t_{0}\right) \int_{0}^{\infty}\left[(1-\beta) \chi_{P}(E)+\sum_{i=1}^{6} \beta_{i} \chi_{i}(E)\right] v\left(E^{\prime}\right) \Sigma_{f}\left(\boldsymbol{r}, E^{\prime}, t\right) f\left(\boldsymbol{r}, E^{\prime}\right) \mathrm{d} E^{\prime} \mathrm{d} E \mathrm{~d}^{3} r
$$

one obtains, after replacing Equation (26) in Equation (25) and the division of the result by $I_{F}$, that

$$
\left(\frac{\Lambda}{f_{D}}\right) \frac{\mathrm{d}^{2} n(t)}{\mathrm{d} t^{2}}+\left(\Lambda+\frac{f_{A} \Lambda}{f_{D}}-\frac{1}{f_{D}}+\frac{\beta}{f_{D}}\right) \frac{\mathrm{d} n(t)}{\mathrm{d} t}+(-\rho+\beta) n(t)-\Lambda \sum_{i=1}^{6} \lambda_{i} C_{i}(t)-\frac{\Lambda}{f_{D}} \sum_{i=1}^{6} \lambda_{i} \frac{\mathrm{d} C_{i}(t)}{\mathrm{d} t}=0
$$

It was considered that the time variation of the diffusion coefficient and of the neutron cross sections are negligible, which immediately implies that their derivatives are null. The several kinetic parameters that appear in Equation (28) are defined as:

$$
\begin{gathered}
\Lambda \equiv \frac{1}{I_{F}} \int_{V} \int_{0}^{\infty} \frac{1}{V} \phi^{*}\left(\boldsymbol{r}, E, t_{0}\right) f(\boldsymbol{r}, E) \mathrm{d} E \mathrm{~d}^{3} r, \\
\frac{1}{f_{D}} \equiv \frac{1}{I_{F} \Lambda} \int_{V}^{\infty} \int_{0}^{\infty} \frac{3 D}{v^{2}} \phi^{*}\left(\boldsymbol{r}, E, t_{0}\right) f(\boldsymbol{r}, E) \mathrm{d} E \mathrm{~d}^{3} r, \\
\rho \equiv-\frac{1}{I_{F}} \int_{V}^{\infty} \int_{0}^{\infty} \phi^{*}\left(\boldsymbol{r}, E, t_{0}\right)\left\{\left(F-F_{0}\right)-\left(L-L_{0}\right)\right\} f(\boldsymbol{r}, E) \mathrm{d} E \mathrm{~d}^{3} r,
\end{gathered}
$$




$$
\begin{gathered}
\beta_{i} \equiv \frac{1}{I_{F}} \int_{V} \int_{0}^{\infty} F_{i} \phi^{*}\left(\boldsymbol{r}, E, t_{0}\right) f(\boldsymbol{r}, E) \mathrm{d} E \mathrm{~d}^{3} r, \\
\beta \equiv \sum_{i=1}^{6} \beta_{i} \\
f_{A} \equiv \frac{1}{\Lambda}+\frac{f_{D}}{\Lambda I_{F}} \int_{V} \int_{0}^{\infty} \frac{3 D}{V} \phi^{*}\left(\boldsymbol{r}, E, t_{0}\right)\{(L-F) f(\boldsymbol{r}, E)\} \mathrm{d} E \mathrm{~d}^{3} r-\frac{1}{\Lambda I_{F}} \sum_{i=1}^{6} \int_{V} \int_{0}^{\infty} \phi^{*}\left(\boldsymbol{r}, E, t_{0}\right) F_{i} f(\boldsymbol{r}, E) \mathrm{d} E \mathrm{~d}^{3} r
\end{gathered}
$$

and

$$
C_{i}(t) \equiv \frac{1}{\Lambda} \int_{V}^{\infty} \int_{0}^{\infty} \chi_{i}(E) \phi^{*}\left(\boldsymbol{r}, E, t_{0}\right) C_{i}(\boldsymbol{r}, t) \mathrm{d} E \mathrm{~d}^{3} r .
$$

In multiplying Equation (10) by the adjoint flux of neutrons and after that integrating in the volume and in the energy $E$, after using Equation (26), one obtains that:

$$
\begin{aligned}
& \int_{V}^{\infty} \int_{0}^{\infty} \chi_{i}(E) \phi^{*}\left(\boldsymbol{r}, E, t_{0}\right) \frac{\partial C_{i}(\boldsymbol{r}, t)}{\partial t} \mathrm{~d} E \mathrm{~d}^{3} r \\
& =n(t) \iint_{V}^{\infty} \phi_{0}^{*}\left(\boldsymbol{r}, E, t_{0}\right) F_{i} f(\boldsymbol{r}, E) \mathrm{d} E \mathrm{~d}^{3} r-\lambda_{i} \iint_{V}^{\infty} \chi_{0}(E) \phi^{*}\left(\boldsymbol{r}, E, t_{0}\right) C_{i}(\boldsymbol{r}, t) \mathrm{d} E \mathrm{~d}^{3} r
\end{aligned}
$$

Dividing Equation (36) by $\Lambda I_{F}$ and using Equations (32) and (35), one obtains:

$$
\frac{\mathrm{d} C_{i}(\boldsymbol{r}, t)}{\mathrm{d} t}=-\lambda_{i} C_{i}(\boldsymbol{r}, t)+\frac{\beta_{i}}{\Lambda} n(t)
$$

where $i=1,2, \cdots, 6$.

Equations (28) and (37) form the new model for the point kinetics, called modified point kinetics.

\section{Equivalency of the Modified Point Kinetics Equations with Respect the Classic Point Kinetics Equations}

The classical point kinetics Equations can be obtained in many ways. Following the development which resulted in the modified point kinetic Equations, the model for the classical point kinetics is obtained by making the approximation known as Fick's Law in Equation (15),

$$
\boldsymbol{J}(\boldsymbol{r}, E, t)=-\frac{1}{3 \Sigma_{T R}(\boldsymbol{r}, E, t)} \nabla \phi(\boldsymbol{r}, E, t)=-D(\boldsymbol{r}, E, t) \nabla \phi(\boldsymbol{r}, E, t) .
$$

From there on, some terms of the first and second derivative of $n(t)$ and the first-order derivative of $C_{i}(t)$ appear in Equation (17) and do not appear in the corresponding equation for the classical kinetics. When $1 / f_{D}(t)$ tends to zero, Equation (28) results to the classical point kinetics equation.

$$
\frac{\mathrm{d} n(t)}{\mathrm{d} t}=\frac{(\rho(t)-\beta)}{\Lambda} n(t)+\sum_{i=1}^{N} \lambda_{i} C_{i}(t) .
$$

The new parameters $f_{D}(t)$ and $f_{A}(t)$ can be called as neutron transport frequency and neutron absorption frequency, respectively. When tends to zero the Equation (28) lies in Equation (39), in other words, the point classical kinetic equation. Equivalent to state that the neutron transport frequency is much larger than the other parameters.

Considering a homogeneous medium Equations (30) and (34) are simplified. First, the diffusion coefficient constant and the speed are considered as the medium is homogeneous, which gives:

$$
\frac{1}{f_{D}} \equiv\left(\frac{3 D}{v \Lambda}\right) \frac{1}{I_{F}} \int_{V}^{\infty} \int_{0}^{\infty}\left(\frac{1}{v}\right) \phi^{*}\left(\boldsymbol{r}, E, t_{0}\right) f(\boldsymbol{r}, E) \mathrm{d} E \mathrm{~d}^{3} r .
$$


Note that appears in the definition of Equation (29) into Equation (40).

$$
\frac{1}{f_{D}} \equiv\left(\frac{3 D}{v \Lambda}\right) \Lambda=\frac{3 D}{v}
$$

Therefore,

$$
f_{D}=\frac{v}{3 D}
$$

In Equation (34) replace the operators $L, F$ and $F_{i}$.

$$
\begin{aligned}
f_{A} \equiv & \frac{1}{\Lambda}+\frac{f_{D}}{\Lambda I_{F}} \int_{V} \int_{0}^{\infty} \frac{3 D}{V} \phi^{*}\left(\boldsymbol{r}, E, t_{0}\right) \Sigma_{t}(\boldsymbol{r}, E, t) f(\boldsymbol{r}, E) \mathrm{d} E \mathrm{~d}^{3} r \\
& -\frac{f_{D}}{\Lambda I_{F}} \int_{V}^{\infty} \int_{0}^{\infty} \int_{0}^{\infty} \Sigma_{S 0}\left(\boldsymbol{r}, E^{\prime} \rightarrow E, t\right)\left(\frac{3 D}{v} \phi^{*}\left(\boldsymbol{r}, E^{\prime}, t_{0}\right) f\left(\boldsymbol{r}, E^{\prime}\right)\right) \mathrm{d} E^{\prime} \mathrm{d} E \mathrm{~d}^{3} r \\
& -\frac{f_{D}}{\Lambda I_{F}} \int_{V}^{\infty} \int_{0}^{\infty} \frac{3 D}{v}(1-\beta) \chi_{f}(E) \int_{0}^{\infty} v\left(E^{\prime}\right) \Sigma_{f}\left(\boldsymbol{r}, E^{\prime}, t\right)\left(\phi^{*}\left(\boldsymbol{r}, E^{\prime}, t_{0}\right) f\left(\boldsymbol{r}, E^{\prime}\right)\right) \mathrm{d} E^{\prime} \mathrm{d} E \mathrm{~d}^{3} r \\
& -\frac{f_{D}}{\Lambda I_{F}} \int_{V}^{\infty} \int_{0}^{\infty} \frac{3 D}{v} \sum_{i=1}^{6} \beta_{i} \chi_{i}(E) \int_{0}^{\infty} v\left(E^{\prime}\right) \Sigma_{f}\left(\boldsymbol{r} E^{\prime}, t\right)\left(\phi^{*}\left(\boldsymbol{r}, E^{\prime}, t_{0}\right) f\left(\boldsymbol{r}, E^{\prime}\right)\right) \mathrm{d} E^{\prime} \mathrm{d} E \mathrm{~d}^{3} r \\
& -\frac{1}{\Lambda I_{F}} \sum_{i=1}^{6} \int_{V}^{\infty} \int_{0}^{\infty} \beta_{i} \chi_{i}(E) \int_{0}^{\infty} v\left(E^{\prime}\right) \Sigma_{f}\left(\boldsymbol{r}, E^{\prime}, t\right)\left(\phi^{*}\left(\boldsymbol{r}, E, t_{0}\right) f(\boldsymbol{r}, E)\right) \mathrm{d}_{i}^{\prime}{\mathrm{d} E \mathrm{~d}^{3} r .} .
\end{aligned}
$$

Considering the homogeneous medium rewrite the Equation (43):

$$
\begin{aligned}
f_{A}= & \frac{1}{\Lambda}+\left(\frac{f_{D}}{\Lambda} \frac{3 D}{v} v \sum_{t}-\frac{f_{D}}{\Lambda} \frac{3 D}{v} v \Sigma_{S 0}\right) \frac{1}{I_{F}} \int_{V}^{\infty} \int_{0}^{\infty} \frac{1}{v} \phi^{*}\left(\boldsymbol{r}, E, t_{0}\right) f(\boldsymbol{r}, E) \mathrm{d} E \mathrm{~d}^{3} r \\
& -\frac{f_{D}}{\Lambda I_{F}} \frac{3 D}{v} \int_{V}^{\infty} \int_{0}^{\infty}\left[(1-\beta) \chi_{f}+2 \sum_{i=1}^{6} \beta_{i} \chi_{i} \int_{0}^{\infty} v\left(E^{\prime}\right) \sum_{f}\left(\boldsymbol{r}, E^{\prime}, t\right) \phi^{*}\left(\boldsymbol{r}, E^{\prime}, t_{0}\right) f\left(\boldsymbol{r}, E^{\prime}\right) \mathrm{d} E^{\prime} \mathrm{d} E \mathrm{~d}^{3} r .\right.
\end{aligned}
$$

Replacing the Equations (27), (29) and (42) in Equation (44):

$$
f_{A}=\frac{1}{\Lambda}+v\left(\Sigma_{t}-\Sigma_{S 0}\right)-\frac{1}{\Lambda}
$$

once:

$$
\Sigma_{t}=\Sigma_{a}+\Sigma_{S 0}
$$

follows:

$$
f_{A}=v \Sigma_{a}
$$

\section{Analytical Solution of the Modified Point Kinetics Equations for One Group of Precursors with Constant Reactivity}

The solution for point kinetics equations can be obtained in several ways, as in [6]-[8]. Point kinetics equations without the approximation related to the time derivative of neutron current density, for one group of precursors and constant reactivity, according to Equations (28) and (37), are:

$$
\frac{1}{f_{D}} \frac{\mathrm{d}^{2} n(t)}{\mathrm{d} t^{2}}+\left(1+\frac{f_{A}}{f_{D}}-\frac{1}{f_{D}} \frac{(1-\beta)}{\Lambda}\right) \frac{\mathrm{d} n(t)}{\mathrm{d} t}=\frac{\left(\rho_{0}-\beta\right)}{\Lambda} n(t)+\lambda C(t)+\frac{\lambda}{f_{D}} \frac{\mathrm{d} C(t)}{\mathrm{d} t}
$$

and 


$$
\frac{\mathrm{d} C(t)}{\mathrm{d} t}=-\lambda C(t)+\frac{\beta}{\Lambda} n(t)
$$

The initial conditions are:

$$
\begin{aligned}
& \left.\frac{\mathrm{d} C(t)}{\mathrm{d} t}\right|_{t=0}=0, \\
& C(0)=\frac{\beta}{\lambda \Lambda} n(0)
\end{aligned}
$$

and

$$
n(0)=n_{0} \text {. }
$$

Applying the conditions (50), (51) and (52) in Equation (48) it is possible to write:

$$
\left.\frac{1}{f_{D}} \frac{\mathrm{d}^{2} n(t)}{\mathrm{d} t^{2}}\right|_{t=0}+\left.\left(1+\frac{f_{\mathrm{A}}}{f_{D}}-\frac{1}{f_{D}} \frac{(1-\beta)}{\Lambda}\right) \frac{\mathrm{d} n(t)}{\mathrm{d} t}\right|_{t=0}=\frac{\rho_{0}}{\Lambda} n_{0} .
$$

One therefore chooses

$$
\left.\frac{\mathrm{d} n(t)}{\mathrm{d} t}\right|_{t=0}=\frac{\rho_{0}}{\Lambda} n_{0} .
$$

It is possible to verify that the initial conditions (50), (51) and (52) as applied to Equation (38), that is, to the classical point kinetics, result exactly in Equation (54). So, in replacing Equation (54) in Equation (53) it results:

$$
\left.\frac{\mathrm{d}^{2} n(t)}{\mathrm{d} t^{2}}\right|_{t=0}=\left(\frac{\rho_{0}(1-\beta)-\rho_{0} f_{A} \Lambda}{\Lambda^{2}}\right) n_{0} .
$$

It adds to the Equation (48) with Equation (49):

$$
\frac{1}{f_{D}} \cdot \frac{\mathrm{d}^{2} n(t)}{\mathrm{d} t^{2}}+\left(1+\frac{f_{A}}{f_{D}}-\frac{1}{f_{D}} \frac{(1-\beta)}{\Lambda}\right) \frac{\mathrm{d} n(t)}{\mathrm{d} t}=\frac{\rho_{0}}{\Lambda} n(t)+\left(\frac{\lambda}{f_{D}}-1\right) \frac{\mathrm{d} C(t)}{\mathrm{d} t}
$$

You can also replace $\mathrm{d} C(t) / \mathrm{d} t$ defined by Equation (49) in Equation (48):

$$
\frac{1}{f_{D}} \cdot \frac{\mathrm{d}^{2} n(t)}{\mathrm{d} t^{2}}+\left(1+\frac{f_{A}}{f_{D}}-\frac{1}{f_{D}} \frac{(1-\beta)}{\Lambda}\right) \frac{\mathrm{d} n(t)}{\mathrm{d} t}=\left\{\frac{\rho_{0}-\beta}{\Lambda}+\frac{\lambda \beta}{\Lambda f_{D}}\right\} n(t)+\left(\lambda-\frac{\lambda^{2}}{f_{D}}\right) C(t) .
$$

The Equation (57) is derived in the time. Then, it results that:

$$
\frac{1}{f_{D}} \cdot \frac{\mathrm{d}^{3} n(t)}{\mathrm{d} t^{3}}+\left(1+\frac{f_{A}}{f_{D}}-\frac{(1-\beta)}{\Lambda \cdot f_{D}}\right) \frac{\mathrm{d}^{2} n(t)}{\mathrm{d} t^{2}}=\left\{\frac{\rho_{0}-\beta}{\Lambda}+\frac{\lambda \beta}{\Lambda f_{D}}\right\} \frac{\mathrm{d} n(t)}{\mathrm{d} t}+\left(\lambda-\frac{\lambda^{2}}{f_{D}}\right) \cdot \frac{\mathrm{d} C(t)}{\mathrm{d} t}
$$

Multiplying the Equation (56) by $\lambda$ :

$$
\frac{\lambda}{f_{D}} \cdot \frac{\mathrm{d}^{2} n(t)}{\mathrm{d} t^{2}}+\left(\lambda+\lambda \frac{f_{A}}{f_{D}}-\frac{\lambda}{f_{D}} \frac{(1-\beta)}{\Lambda}\right) \frac{\mathrm{d} n(t)}{\mathrm{d} t}=\frac{\lambda \rho_{0}}{\Lambda} n(t)+\left(\frac{\lambda^{2}}{f_{D}}-\lambda\right) \frac{\mathrm{d} C(t)}{\mathrm{d} t} .
$$

It adds to the Equation (58) with the Equation (59):

$$
\begin{aligned}
& \frac{1}{f_{D}} \cdot \frac{\mathrm{d}^{3} n(t)}{\mathrm{d} t^{3}}+\left\{1+\frac{f_{A}}{f_{D}}-\frac{(1-\beta)}{\Lambda \cdot f_{D}}-\frac{\lambda}{f_{D}}\right\} \frac{\mathrm{d}^{2} n(t)}{\mathrm{d} t^{2}} \\
& =\left\{\frac{\rho_{0}-\beta}{\Lambda}+\frac{2 \lambda \beta}{\Lambda f_{D}}+\lambda+\lambda \frac{f_{A}}{f_{D}}-\frac{\lambda}{f_{D} \Lambda}\right\} \frac{\mathrm{d} n(t)}{\mathrm{d} t}+\frac{\lambda \rho(t)}{\Lambda} n(t)
\end{aligned}
$$

The Equation (60) is a third-order homogeneous linear differential equation and will be solved by the Laplace Transform technique. One can simplify Equation (60) as: 


$$
\begin{aligned}
& \frac{1}{f_{D}} \frac{\mathrm{d}^{3} n(t)}{\mathrm{d} t^{3}}+\left\{1+\frac{f_{A}}{f_{D}}-\frac{(1-\beta)}{\Lambda f_{D}}-\frac{\lambda}{f_{D}}\right\} \frac{\mathrm{d}^{2} n(t)}{\mathrm{d} t^{2}} \\
& -\left\{\frac{\rho_{0}-\beta}{\Lambda}+\frac{2 \lambda \beta}{\Lambda f_{D}}+\lambda+\lambda \frac{f_{A}}{f_{D}}-\frac{\lambda}{f_{D} \Lambda}\right\} \frac{\mathrm{d} n(t)}{\mathrm{d} t}-\frac{\lambda \rho(t)}{\Lambda} n(t)=0
\end{aligned}
$$

In applying the Laplace transform [9] in Equation (61), it results that:

$$
\begin{aligned}
& \frac{1}{f_{D}}\left\{s^{3} \mathcal{L}(n(t))-s^{2} n(0)-s D(n(0))-D^{2}(n(0))\right\}+\left\{1+\frac{f_{A}}{f_{D}}-\frac{(1-\beta)}{\Lambda f_{D}}-\frac{\lambda}{f_{D}}\right\}\left\{s^{2} \mathcal{L}(n(t))-s n(0)-D(n(0))\right\} \\
& -\left\{\frac{\rho_{0}-\beta}{\Lambda}+\frac{2 \lambda \beta}{\Lambda f_{D}}+\lambda+\lambda \frac{f_{A}}{f_{D}}-\frac{\lambda}{f_{D} \Lambda}\right\}\{s \mathcal{L}(n(t))-n(0)\}-\frac{\lambda \rho_{0}}{\Lambda} \mathcal{L}(n(t))=0,
\end{aligned}
$$

being,

$$
A \equiv\left\{1+\frac{f_{A}}{f_{D}}-\frac{(1-\beta)}{\Lambda f_{D}}-\frac{\lambda}{f_{D}}\right\}
$$

and

$$
B \equiv\left\{\frac{\rho_{0}-\beta}{\Lambda}+\frac{2 \lambda \beta}{\Lambda f_{D}}+\lambda+\lambda \frac{f_{A}}{f_{D}}-\frac{\lambda}{f_{D} \Lambda}\right\}
$$

In Equation (62) we isolate the Laplace transform of the neutron density:

$$
\mathcal{L}(n(t))=\frac{\left\{\left(\frac{1}{f_{D}} n(0)\right) s^{2}+\left(\frac{1}{f_{D}} D(n(0))+A n(0)\right) s+\left(\frac{1}{f_{D}} D^{2}(n(0))+A D(n(0))+B n(0)\right)\right\}}{\frac{1}{f_{D}} s^{3}+A s^{2}+B s+\frac{\lambda \rho_{0}}{\Lambda}}
$$

Applying the reverse Laplace transform on both sides of Equation (65) one have that:

$$
n(t)=\mathcal{L}^{-1}\left(\frac{\left\{\left(\frac{1}{f_{D}} n(0)\right) s^{2}+\left(\frac{1}{f_{D}} D(n(0))+A n(0)\right) s+\left(\frac{1}{f_{D}} D^{2}(n(0))+A D(n(0))+B n(0)\right)\right\}}{\frac{1}{f_{D}} s^{3}+A s^{2}+B s+\frac{\lambda \rho_{0}}{\Lambda}}\right)
$$

To solve the reverse Laplace transform one should factor the polynomials so to obtain:

$$
n(t)=\mathcal{L}^{-1}\left(\frac{K_{1}}{s-\mu_{1}}+\frac{K_{2}}{s-\mu_{2}}+\frac{K_{3}}{s-\mu_{3}}\right)
$$

Note that the Equation (67) corresponds exactly to Equation (66), in other words, can rewrite (67):

$$
n(t)=\mathcal{L}^{-1}\left(\frac{\left(K_{1}+K_{2}+K_{3}\right) s^{2}-\left(K_{1}\left(\mu_{2}+\mu_{3}\right)+K_{2}\left(\mu_{1}+\mu_{3}\right)+K_{3}\left(\mu_{1}+\mu_{2}\right)\right) s+\mu_{2} \mu_{3} K_{1}+\mu_{1} \mu_{3} K_{2}+\mu_{1} \mu_{2} K_{3}}{s^{3}-\left(\mu_{1}+\mu_{2}+\mu_{3}\right) s^{2}+\left(\mu_{1} \mu_{2}+\mu_{2} \mu_{3}+\mu_{1} \mu_{3}\right) s-\mu_{1} \mu_{2} \mu_{3}}\right)
$$

Comparing the above equations it is possible to deduce the number system:

$$
\begin{aligned}
K_{1}= & n(0)-\frac{\left(D(n(0))+f_{D} A \cdot n(0)+n(0) \cdot\left(\mu_{2}+\mu_{3}\right)\right)}{\left(\mu_{2}-\mu_{1}\right)} \\
& +\frac{\left[n(0)\left(\mu_{3}\right)^{2}+\left(D(n(0))+f_{D} A \cdot n(0)\right) \mu_{3}+D^{2}(n(0))+f_{D} A \cdot D(n(0))+f_{D} B \cdot n(0)\right] \cdot\left(\mu_{3}-\mu_{2}\right)}{\mu_{1}\left(\mu_{2}\right)^{2}-\mu_{1}\left(\mu_{3}\right)^{2}+\mu_{2}\left(\mu_{3}\right)^{2}-\mu_{2}\left(\mu_{1}\right)^{2}+\mu_{3}\left(\mu_{1}\right)^{2}-\mu_{3}\left(\mu_{2}\right)^{2}},
\end{aligned}
$$




$$
\begin{gathered}
K_{2}=\frac{\left(D(n(0))+f_{D} A \cdot n(0)+n(0) \cdot\left(\mu_{2}+\mu_{3}\right)\right)}{\left(\mu_{2}-\mu_{1}\right)} \\
+\frac{\left[n(0)\left(\mu_{3}\right)^{2}+\left(D(n(0))+f_{D} A \cdot n(0)\right) \mu_{3}+D^{2}(n(0))+f_{D} A \cdot D(n(0))+f_{D} B \cdot n(0)\right] \cdot\left(\mu_{1}-\mu_{3}\right)}{\mu_{1}\left(\mu_{2}\right)^{2}-\mu_{1}\left(\mu_{3}\right)^{2}+\mu_{2}\left(\mu_{3}\right)^{2}-\mu_{2}\left(\mu_{1}\right)^{2}+\mu_{3}\left(\mu_{1}\right)^{2}-\mu_{3}\left(\mu_{2}\right)^{2}}, \\
K_{3}=\frac{\left[n(0)\left(\mu_{3}\right)^{2}+\left(D(n(0))+f_{D} A \cdot n(0)\right) \mu_{3}+D^{2}(n(0))+f_{D} A \cdot D(n(0))+f_{D} B \cdot n(0)\right] \cdot\left(\mu_{2}-\mu_{1}\right)}{\mu_{1}\left(\mu_{2}\right)^{2}-\mu_{1}\left(\mu_{3}\right)^{2}+\mu_{2}\left(\mu_{3}\right)^{2}-\mu_{2}\left(\mu_{1}\right)^{2}+\mu_{3}\left(\mu_{1}\right)^{2}-\mu_{3}\left(\mu_{2}\right)^{2}} \\
\mu_{1}=-f_{D} A-\mu_{2}-\mu_{3} \\
\mu_{3}\left(\mu_{2}\right)^{2}+\left(f_{D} A \cdot \mu_{3}+\left(\mu_{3}\right)^{2}\right) \mu_{2}-\frac{f_{D} \lambda \rho_{0}}{\Lambda}=0
\end{gathered}
$$

and

$$
\left(\mu_{3}\right)^{3}+f_{D} A \cdot\left(\mu_{3}\right)^{2}+\left(f_{D} B\right) \mu_{3}+\frac{f_{D} \lambda \rho_{0}}{\Lambda}=0
$$

The Equation (73) can be solved directly to obtain the value of $\mu_{3}$. Note that it is an equation of the third degree. Initially it replaces the variable $\mu_{3}$ by $x-\frac{f_{D} \cdot A}{3}$ :

$$
\begin{array}{r}
x^{3}+\left(-\frac{1}{3}\left(f_{D} \cdot A\right)^{2}+f_{D} B\right) x-\frac{\left(f_{D}\right)^{2} \cdot A \cdot B}{3} \\
-\frac{1}{27} \cdot\left(f_{D} A\right)^{3}+\frac{1}{9} \cdot\left(f_{D} A\right)^{2}+\frac{f_{D} \lambda \rho_{0}}{\Lambda}=0
\end{array}
$$

Note that in Equation (74) the term corresponding to $x^{2}$ is reset. Thus, it can be rewritten:

$$
x^{3}+p \cdot x+q=0
$$

The Equation (75) was resolved according [10] and the solution is known as Cardano-Tartaglia's formula:

$$
x=x_{1}=\sqrt[3]{-\frac{q}{2}+\sqrt{\left(\frac{q}{2}\right)^{2}+\left(\frac{p}{3}\right)^{3}}}+\sqrt[3]{-\frac{q}{2}-\sqrt{\left(\frac{q}{2}\right)^{2}+\left(\frac{p}{3}\right)^{3}}}
$$

Note that Equation (76) corresponds only one of the three solutions of the Equation (85). The other two solutions can be obtained by dividing Equation (75) by $\left(x-x_{1}\right)$ which allow us to obtain:

$$
\frac{x^{3}+p \cdot x+q}{x-x_{1}}=a x^{2}+b \cdot x+c
$$

therefore,

$$
x^{3}+p \cdot x+q=\left(a x^{2}+b \cdot x+c\right)\left(x-x_{1}\right)=0
$$

Follows:

$$
a x^{2}+b \cdot x+c=0
$$

The roots of Equation (77) are the other two solutions of Equation (76). Thus three possible solutions which satisfy the Equation (73) are obtained. The solutions are substituted in Equation (72). Equation (72) becomes an Equation of the second degree variable and can be trivially solved. With solutions and can be obtained from Equation (71). Equations (68), (69) and (70) are all expressed only in terms of, and and so the values of $K_{1}$, $K_{2}$ and $K_{3}$ are determined directly. From the resolution of the inverse Laplace transform of Equation (67), results that: 


$$
n(t)=K_{1} \exp \left(\mu_{1} t\right)+K_{2} \exp \left(\mu_{2} t\right)+K_{3} \exp \left(\mu_{3} t\right)
$$

\section{Results}

Modified point kinetics equations for one group of precursors with constant reactivity are solved with the method described in Section 3. Numerical values for the nuclear parameters are considered from references found in the literature, i.e., [1] [11]-[15] as listed in Table 1.

The values considered in Table 1 for the absorption cross section and the diffusion coefficient correspond to the average cross sections of a typical core in a PWR nuclear reactor, according to [1] and [12].

The initial conditions (50), (51), (52) are used in the calculations of classical and modified equations and the initial conditions (54) and (55) are used only in the modified point kinetics equations. We consider and the values of Table 1. The results are shown in the Figures 1-6 and in the Table 2 to Table 3.

Figures 1-6 show the results of the calculations made with the solution of classical point kinetics equation, modified point kinetics equation with transport frequency equal to $104 \mathrm{~s}^{-1}$ and modified point kinetics equation with transport frequency equal to $103 \mathrm{~s}^{-1}$. In the first three figures the time interval is from 0 to $100 \mathrm{~s}$ and in the last three it goes from 0 to $10 \mathrm{~s}$. Note that in the graph contained in Figure 3 the order of magnitude for neutron density of the classical kinetics and of the modified kinetics are quite different for a reactivity of 0.007 .

The variation in the neutron density as a function of the reactivity is seen through a comparison between the graphs. It is possible to see that, for a reactivity equal to the fraction of neutrons delayed by the total of neutrons, the neutron density obtained by the classical point kinetics equations for a time corresponding to $100 \mathrm{~s}$ is of the

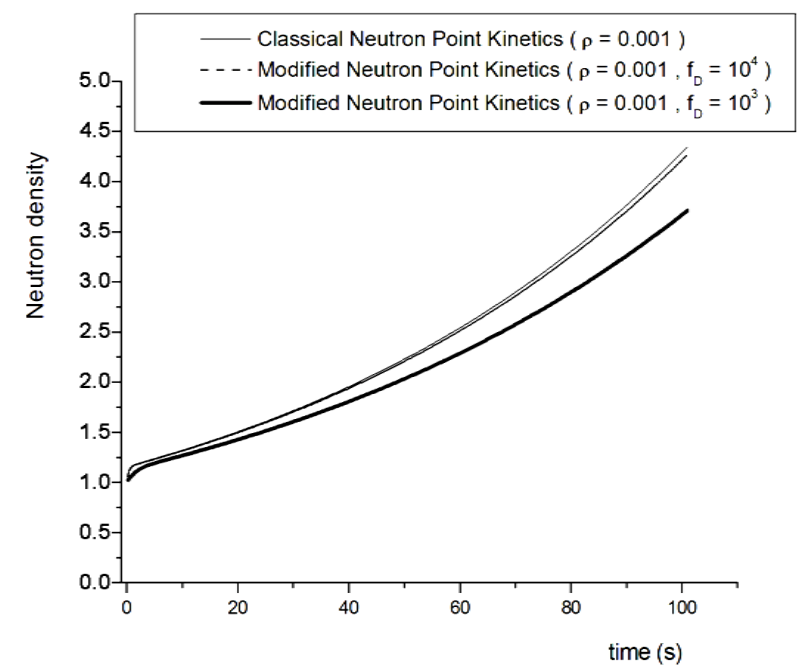

Figure 1. Neutron density as a function of the time of $0 \mathrm{~s}$ at $100 \mathrm{~s}$ for a reactivity of 0.001 .

Table 1. Parameters used in the tests.

\begin{tabular}{|c|c|c|}
\hline Parameter & Symbol & Value \\
\hline Decay constant & $\lambda$ & $0.0810958 \mathrm{~s}^{-1}$ \\
\hline Mean generation time & $\Lambda$ & $0.002 \mathrm{~s}$ \\
\hline Absorption cross section & $\Sigma_{a}$ & $0.14 \mathrm{~cm}^{-1}$ \\
\hline Diffusion coefficient & $D$ & $10 \mathrm{~cm}$ \\
\hline Neutron velocity & $v$ & $3 \times 10^{6} \mathrm{~cm} / \mathrm{s}$ \\
\hline Fraction of delayed neutrons & $\beta$ & 0.007 \\
\hline Absorption frequency & $f_{A}$ & $4.167 \mathrm{~s}^{-1}$ \\
\hline
\end{tabular}


Table 2. Calculation of $\mathrm{n}(\mathrm{t})\left(\mathrm{cm}^{-3}\right)$ with point kinetics for a group of precursors with a neutron transport frequency of $10^{4} \mathrm{~s}^{-1}$.

\begin{tabular}{|c|c|c|c|c|c|c|}
\hline Model and Reactivity & $\mathrm{t}=0.4 \mathrm{~s}$ & $t=1 \mathrm{~s}$ & $t=10 s$ & $t=20 s$ & $\mathrm{t}=40 \mathrm{~s}$ & $t=100 s$ \\
\hline \multicolumn{7}{|l|}{ Classical kinetics } \\
\hline$\rho=0.001$ & 1.1173 & 1.1648 & 1.3186 & 1.5033 & 1.9537 & 4.2888 \\
\hline \multicolumn{7}{|l|}{ Modified Kinetics } \\
\hline$\rho=0.001$ & 1.0979 & 1.1528 & 1.3129 & 1.4946 & 1.9368 & 4.2151 \\
\hline \multicolumn{7}{|l|}{ Classical kinetics } \\
\hline$\rho=0.003$ & 1.4161 & 1.6770 & 2.9278 & 5.1718 & 16.138 & 490.25 \\
\hline \multicolumn{7}{|l|}{ Modified Kinetics } \\
\hline$\rho=0.003$ & 1.3341 & 1.5954 & 2.8417 & 4.9561 & 15.076 & 424.36 \\
\hline \multicolumn{7}{|l|}{ Classical kinetics } \\
\hline$\rho=0.006$ & 2.0958 & 3.4712 & 56.554 & 947.92 & $2.663 \times 10^{5}$ & $5.905 \times 10^{12}$ \\
\hline \multicolumn{7}{|l|}{ Modified Kinetics } \\
\hline$\rho=0.006$ & 1.8212 & 2.9008 & 38.432 & 487.09 & 78236 & $3.242 \times 10^{11}$ \\
\hline
\end{tabular}

Table 3. Calculation of $\mathrm{n}(\mathrm{t})\left(\mathrm{cm}^{-3}\right)$ with point kinetics for a group of precursors with a neutron transport frequency of $10^{3} \mathrm{~s}^{-1}$.

\begin{tabular}{|c|c|c|c|c|c|c|}
\hline Model and Reactivity & $\mathrm{t}=0.4 \mathrm{~s}$ & $t=1 s$ & $t=10 s$ & $t=20 s$ & $t=40 s$ & $t=100 s$ \\
\hline \multicolumn{7}{|l|}{ Classical kinetics } \\
\hline$\rho=0.001$ & 1.1173 & 1.1648 & 1.3186 & 1.5033 & 1.9537 & 4.2888 \\
\hline \multicolumn{7}{|l|}{ Modified kinetics } \\
\hline$\rho=0.001$ & 1.0379 & 1.0798 & 1.2688 & 1.4279 & 1.8083 & 3.6727 \\
\hline \multicolumn{7}{|l|}{ Classical kinetics } \\
\hline$\rho=0.003$ & 1.4161 & 1.6770 & 2.9278 & 5.1718 & 16.138 & 490.25 \\
\hline \multicolumn{7}{|l|}{ Modified kinetics } \\
\hline$\rho=0.003$ & 1.1185 & 1.2640 & 2.3225 & 3.7127 & 9.4734 & 157.38 \\
\hline \multicolumn{7}{|l|}{ Classical kinetics } \\
\hline$\rho=0.006$ & 2.0958 & 3.4712 & 56.554 & 947.92 & $2.663 \times 10^{5}$ & $5.905 \times 10^{12}$ \\
\hline \multicolumn{7}{|l|}{ Modified kinetics } \\
\hline$\rho=0.006$ & 1.2524 & 1.6157 & 9.1475 & 42.311 & 898.86 & $8.617 \times 10^{6}$ \\
\hline
\end{tabular}

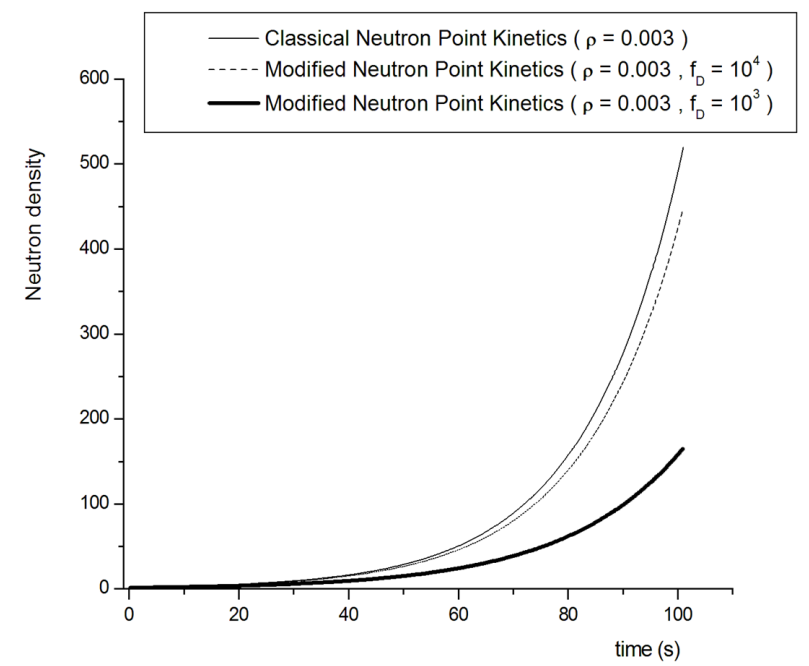

Figure 2. Neutron density as a function of the time of $0 \mathrm{~s}$ at $100 \mathrm{~s}$ for a reactivity of 0.003 . 


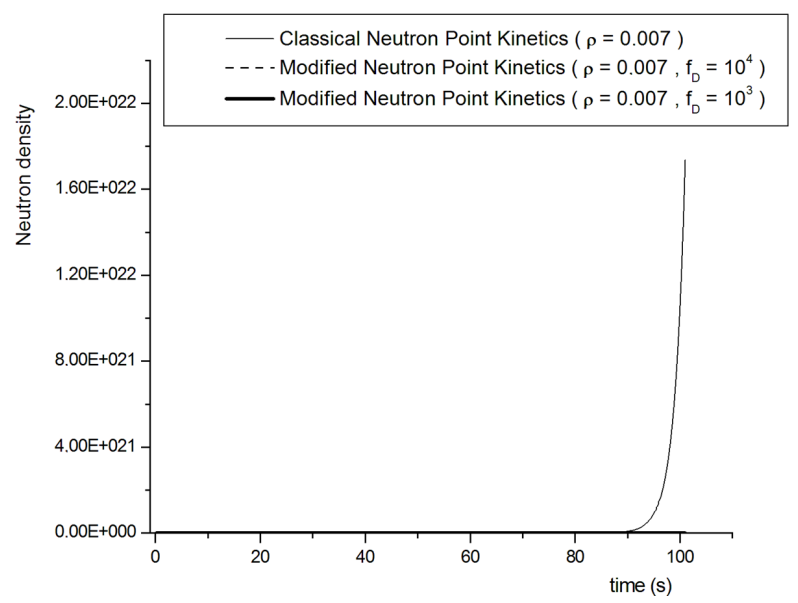

Figure 3. Neutron density as a function of the time of $0 \mathrm{~s}$ at $40 \mathrm{~s}$ for a reactivity of 0.006 .

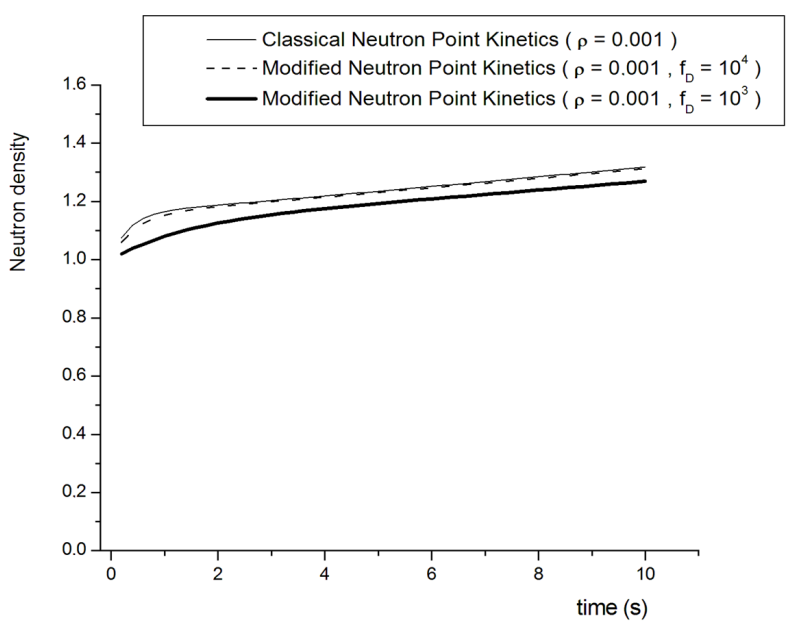

Figure 4. Neutron density as a function of the time of $0 \mathrm{~s}$ at $10 \mathrm{~s}$ for a reactivity of 0.001 .

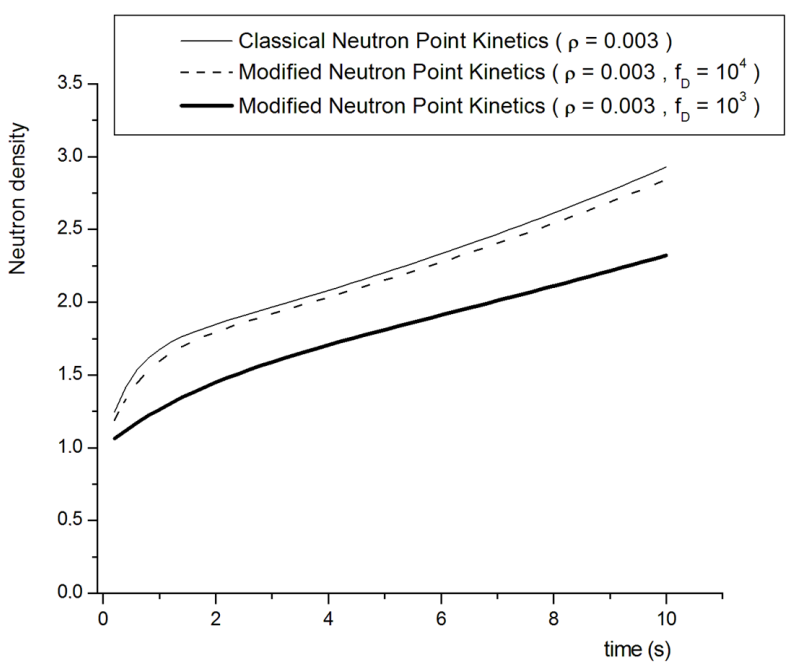

Figure 5. Neutron density as a function of the time of $0 \mathrm{~s}$ at $10 \mathrm{~s}$ for a reactivity of 0.003 . 


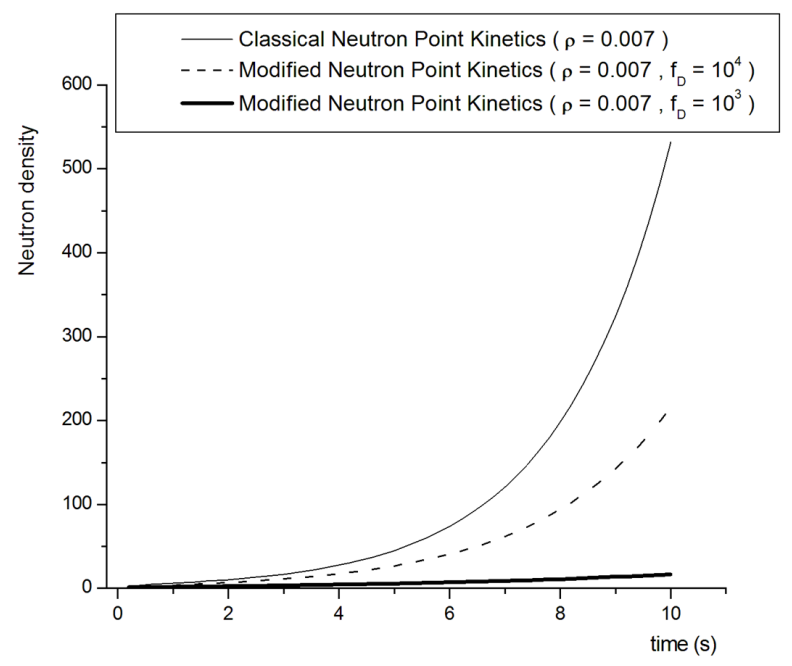

Figure 6. Neutron density as a function of the time of $0 \mathrm{~s}$ at $10 \mathrm{~s}$ for a reactivity of 0.001 .

order of 1022, whilst the use of the modified point kinetics equations is of the order of 109, for a neutron transport frequency of $103 \mathrm{~s}^{-1}$. Thus, the difference between the results of the models is more significant for highreactivity situations.

\section{Conclusions}

The objective of this paper is to obtain a new system of equations called equations of point kinetics modified in which is considered the effect of the time derivative for neutron current density in the Equation (15). In general, the time derivative of the density of neutrons is neglected for the obtainment of the classical model.

The results presented in this article show that the difference between the neutron density obtained from classical point kinetics equations and that obtained from modified point kinetics equations is relevant. With the neutron transport frequency equal to $104 \mathrm{~s}^{-1}$ the difference between the neutron density obtained from classical point kinetics equations and that obtained from point kinetics equations without the approximation for the time derivative of neutron current density is relevant. With a neutron transport frequency equal to $103 \mathrm{~s}^{-1}$ the difference between them it is quite significative.

Modified point kinetics equations imply a significant difference in results, in relation to those obtained with the classical point kinetics. Table 1 and Table 2 show that the results from the classical kinetics have an important difference in relation to the model of the modified point kinetics that increases when the frequency is smaller.

\section{References}

[1] Duderstadt, J.J. and Hamilton, L.J. (1976) Nuclear Reactor Analysis. John Wiley \& Sons Ltd., New York.

[2] Bell, G.I. and Glasstone (1970) Nuclear Reactor Theory. Van Nostrand Reinhold Ltd., New York.

[3] Henry, A.F. (1975) Nuclear Reactor Analysis. The MIT Press, Cambridge and London.

[4] Heizler, S.I. (2010) Asymptotic Telegrapher's Equation (P1) Approximation for the Transport Equation. Nuclear Science and Engineering, 166, 17-35. http://physics.biu.ac.il/files/physics/shared/staff/u47/nse_166_17.pdf

[5] Espinosa-Paredes, G., Polo-Labarrios, M.A., Espinosa-Martinez, E.G. and del Valle-Gallegos, E. (2011) Fractional Neutron Point Kinetics Equations for Nuclear Reactor Dynamics. Annals of Nuclear Energy, 38, 307-330. http://www.sciencedirect.com/science/article/pii/S0306454910003816 http://dx.doi.org/10.1016/j.anucene.2010.10.012

[6] Akcasu, Z., Lellouche, G. and Shotkin, L.M. (1971) Mathematical Methods in Nuclear Reactor Dynamics. Academic Press, New York and London.

[7] Chao, Y.A. and Attard, A. (1985) A Resolution of the Stiffness Problem of Reactor Kinetics. Nuclear Science and En- 
gineering, 90, 40-46. http://www.ans.org/pubs/journals/nse/a_17429 http://dx.doi.org/10.13182/NSE85-7

[8] Zhang, F., Chen, W.Z. and Gui, X.W. (2008) Analytic Method Study of Point-Reactor Kinetic Equation When Cold Start-Up. Annals of Nuclear Energy, 35, 746-749.

http://www.sciencedirect.com/science/article/pii/S0306454907002368 http://dx.doi.org/10.1016/j.anucene.2007.08.015

[9] Hoogenboom, J.E. (1985) The Laplace Transformation of Adjoint Transport Equations. Annals of Nuclear Energy, 12, 151-152. http://www.sciencedirect.com/science/article/pii/030645498590091X http://dx.doi.org/10.1016/0306-4549(85)90091-X

[10] Fuchs, D. and Tabachnikov, S. (2000) Mathematical Omnibus: Thirty Lectures on Classic Mathematics. American Mathematical Society, Rhode Island.

[11] Hetrick, D.L. (1971) Dynamics of Nuclear Reactor. The University of Chicago Press Ltd., Chicago and London.

[12] Stacey, W.M. (2007) Nuclear Reactor Analysis. 2nd Edition, Wiley-VCH GmbH \& CO KGaA, Weinheim.

[13] Kinard, M. and Allen, E.J. (2003) Efficient Numerical Solution of the Point Kinetics Equations in Nuclear Reactor Dynamics. Annals of Nuclear Energy, 31, 1039-1051. http://www.sciencedirect.com/science/article/pii/S0306454904000027 http://dx.doi.org/10.1016/j.anucene.2003.12.008

[14] Palma, D.A.P., Martinez, A.S. and Gonçalves, A.C. (2009) Analytical Solution of Point Kinetics Equations for Linear Reactivity Variation. Annals of Nuclear Energy, 36, 1469-1471. http://www.sciencedirect.com/science/article/pii/S0306454909001947 http://dx.doi.org/10.1016/j.anucene.2009.06.016

[15] Jahanbin, A. and Malmir, H. (2012) Kinetic Parameters Evaluation of PWRs Using Static Cell and Core Calculation Codes. Annals of Nuclear Energy, 41, 110-114. http://www.sciencedirect.com/science/article/pii/S0306454911004464 
Scientific Research Publishing (SCIRP) is one of the largest Open Access journal publishers. It is currently publishing more than 200 open access, online, peer-reviewed journals covering a wide range of academic disciplines. SCIRP serves the worldwide academic communities and contributes to the progress and application of science with its publication.

Other selected journals from SCIRP are listed as below. Submit your manuscript to us via either submit@scirp.org or Online Submission Portal.
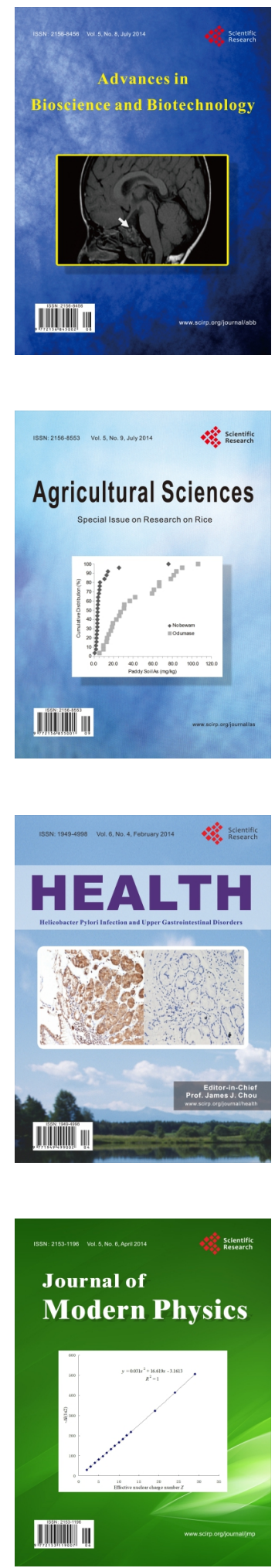
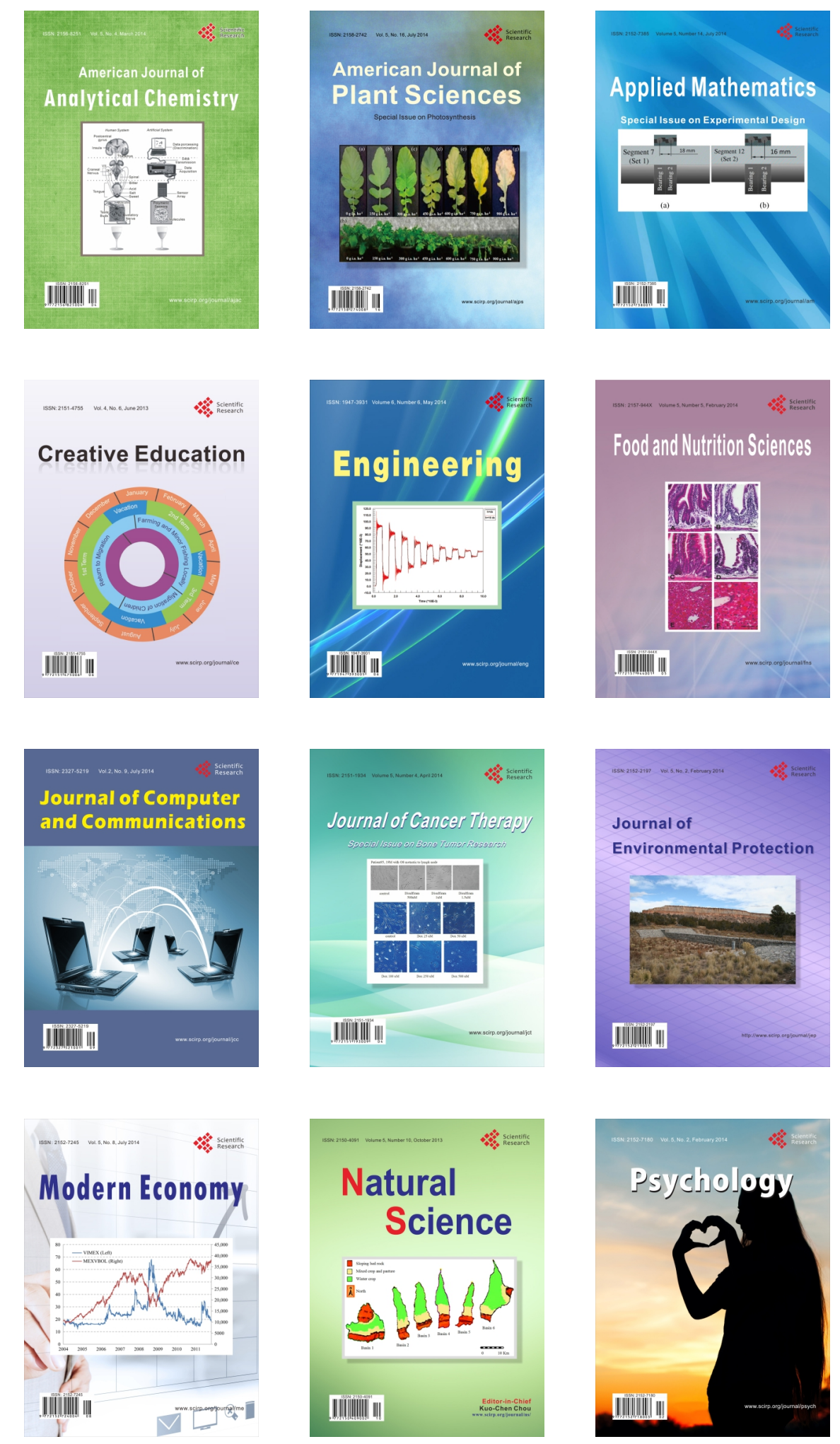